\title{
UNDERSTANDING ADOPTION FACTORS OF OVER-THE-TOP VIDEO SERVICES AMONG MILLENNIAL CONSUMERS
}

\author{
Dr. Sabyasachi Dasgupta \\ Assistant Professor, O.P. Jindal Global University, India \\ Dr. Priya Grover \\ Head-Research \& Development, \\ Brand Mavs (Formerly Known as Brand Mavericks LLP) Kharadi, Pune
}

\begin{abstract}
With growing digitization, the challenge for marketers is to understand how consumers consuming Over-The -Top (OTT) content adopt and consume messages in this format effectively. Superimposing the theoretical framework of Uses and Gratification meant for television to internet platform, this paper in a novel approach tries to understand the consumption patterns and adoptability factors of OTT among consumers. The qualitative methodology adopted for this research, brought out four themes enabling the success of this platform: Convenience, mobility, content and subscription strategies. These strategic parameters will ensure higher engagement levels of the consumers for the OTT content.
\end{abstract}

Key words: Content, Consumer, Subscription, Mobility, Convenience, Digital, OverThe-Top, Adoption, Video, Internet, Millennial, Engagement, Multi-Media, Innovation.

Cite this Article: Dr. Sabyasachi Dasgupta and Dr. Priya Grover, Understanding Adoption Factors of Over-the-Top Video Services among Millennial Consumers, International Journal of Computer Engineering and Technology, 10(1), 2019, pp. 6171.

http://iaeme.com/Home/issue/IJCET?Volume=10\&Issue=1

\section{INTRODUCTION}

Over-The-Top (OTT) content is becoming a big buzzword in today's technology and systems enabled environment. Being one of the largest economies in the world, Indian television industry is going to garner revenues of more than Rs. 975 Billion by 2019. (KPMG-FICCI, 2015). With the full force attempts to increase the nationwide internet (1) penetration, India is likely to witness more than 500 million Internet users by June 2018. (IAMAI, 2017). In this digital era, Over The Top (OTT) content has emerged among millennial as the new standalone digital multi-media platform where multi-cable or direct broadcast satellite television do not distribute or control content, leading to media and consumer autonomy of consumption. 
Viewers will now have complete autonomy in terms of choosing their own content and pricing, keeping in mind the exact type and amount of content they want to view. Another major trend emerging in television content viewing is the emergence of OTT platforms by individual media houses. This trend is primarily fuelled by increase in the number of devices that support these platforms along with tremendous rise in the number of internet and mobile data subscribers. The rise of OTT platforms has also seen growth with the steady but positive increase in the number of people who are wanting and demanding both 'Produced-for-TV' as well as exclusive freshly brewed content on demand anytime of the day, thus making it one of the biggest opportunities in the video and content sector in the entertainment industry. Currently, India has more than 11 OTT platforms with the major one's being HotStar by Star Network, VooT by Viacom18, Sony Liv by Sony, Spuul, Netflix, Eros Now, and Hooq. There are other multiple independent content creators like TVF who are now coming up with their individual OTT platforms like TVF Play in order to compete directly with the mainline platforms by providing freshly brewed content over the others. Thus it is becoming evident that OTT contents and the platforms associated with it are becoming major sources of communication and revenue streams for corporate organizations.

With large OTT services going extremely heavy on marketing for acquiring and grabbing customer's eyeballs, the Video-On-Demand (VOD) market is on the brink of a massive takeoff. Factors like digitization, reduced mobile data costs, high speed internet, and multiscreen penetration across geographies and classes and fixed line broadband growth, forecast huge potential in the sector of OTT services in India, which is likely to grow manifold in the coming years. A trend of people viewing multiple content channels at the same time, along with demand for newer and more exclusive content has led to many media houses and partners to start their own OTT platforms. Although the revenue and business model of the platform remains the same as a traditional TV channels, that is paid advertising, the feasibility of the same has been questioned in multiple forums. Many platforms have however claimed that the traction that is generated by the free content usually helps garner higher paying subscription base. Thus a research in this field would open up multiple opportunities for varied platforms and enable marketers to understand and better their strategy regarding the same.

The aim of this research is to understand and analyze the type of content viewed, expected and demanded by the intended audience, thereby inferring the ways in which consumers take decisions regarding contents in OTT platforms. The objective of this research is to understand gratification factors associated with the adoption and consumption of OTT content.

\section{LITERATURE REVIEW}

There has been a tremendous rise in media consumption in digital formats across the world. The consumers today have the liberty to access the media content of his choice anytime anywhere. This has been possible due to increase in the number of devices supporting digital media and enhanced internet speed. The rise of Netflix, Hulu, Amazon, Apple TV etc. have started challenging the supremacy of the traditional television. (Deloitte, 2017). The Smartphone mobile traffic in 2014 was $1.73 \mathrm{~EB}$ and is projected to grow at a compound rate of $60 \%$ from 2014 to 2019 . (Deloitte, 2017). The digital media worldwide is projected to increase to $\$ 118$ bn by 2021 with a compound annual growth rate of $11 \%$ between 2016 and 2021. (Lacy, 2017).Keeping in line with the global trends, India has also shown an increase in the consumption of all types of content ranging from text, audio or video on different digital platforms. India has become the second largest online market after china with over 460 million internet users. By 2021, it will jump to 635.8 million users. (Statista) A lot of favorable factors like improved networks, better access to internet, multimedia service- 
capable mobile devices and application development ecosystem have contributed to a tremendous increase in media consumption on digital platforms. India has around 300,000 app developers and is the second largest app developer after US. (Deloitte, 2017).

An increase in the usage of smartphones in India has stirred a new era of video consumption on the personal media device. The penetration of smartphones in India is projected to grow to $520 \mathrm{mn}$ by 2020 and broadband penetration will increase from $14 \%$ currently to $40 \%$ in 2020 . (Ernst \& Young, 2016). This rise will be instrumental in a drastic shift of media consumption from traditional to digital. There has been a decline in the percentage of Indian Consumers who prefer watching shows on TV from $47 \%$ to $10 \%$ over the last one year. (Accenture, 2015).

While TV shows are watched on laptops and desktops, smartphones are used to watch short video clips.

So, there is a tremendous shift from the traditional TV viewing to digital viewership and a lot of factors have contributed to this shift. Mobile handsets have become more affordable and technologically advanced. Indian government reforms and promotions to move towards digital. Companies like Facebook, You tube, Hotstar, Saavn, Buzzfeed and so on are increasing their share of investments. Commerce is shifting from offline to online. Internet of Things will soon be a household concept. All these major trends and shifts will further drive people to spend more time on digital mediums.

The global mobile traffic is expected to increase nearly sevenfold from 2016 to 2021 . As per the data available till February 2017, mobile devices accounted for $49.74 \%$ of web page views worldwide with Asia and Africa leading the pack. (Statista)

In India, 59\% consumers watch advertisements in digital media than TV commercials. (Deloitte, 2017). The share of digital advertising in India is projected to double in the next 3 years from $12 \%$ currently to $24 \%$ in 2020 . As per the data available till January 2017 , mobile devices accounted for $79 \%$ of web page views in India. (Statista).Internet access is mainstreaming among Indian consumers and the use of mobile is intensifying. More people spend more time online using a variety of digital tools and sites for content consumption. India at present is the third biggest country in terms of internet users in the world with as many as 121 million Indians accessing internet. There has been $25 \%$ growth in Indian internet users over a year. $59 \%$ of the Indians access the internet via their mobiles. The average time spent on social media is 3 hours and $70 \%$ of Internet users watch online videos. (Statista).An average mobile web user in India consumers 6.2 hours of media daily which includes 102 minutes of mobile media and 79 minutes of online media consumption. Social media and entertainment including both music and video. $21 \%$ of the total time is spent by youth on audio and video entertainment. (Deloitte, 2017)

Among the Social media platforms, Facebook and Twitter account for $47 \%$ and $42 \%$ of overall videos watched online. The remaining $11 \%$ belong to OTT services like Hotstar, Netflix, Amazon Prime video, Jio TV and VOOT. There are 24 million OTT users in India. The market for OTT subscription is projected to grow to 4,000 crore by 2020.Currently, the average amount spent monthly for paid content is Rs.255. (Video OTT vs DTH players: How Netflix, Amazon Prime, Hotstar cornered Indian market, 2017)

OTT or "Over-the-top" refers to video content streamed through internet and not through broadcast or cable television. It includes subscription based services, free, ad supported services and pay-to-watch streams offered by services including iTunes and Video on demand. (Deloitte, 2017)

OTT services are either offered for free or at a small monthly subscription fee. Examples of OTT devices include Smart TV's like Google TV and LG Electronics Channel Plus, 
various gaming devices like Xbox One, PlayStation, smart set-top boxes like Apple TV, tablets and smart phones like iPhones and Android phones. OTT platforms have thus challenged the traditional content and service providers.

Various factors are driving paid subscriptions like need for high quality videos, better viewing experience, ease of access and wider content choice. Though the internet speed in India is one third of the global average, but the optimal data speed along with friendlier data costs would contribute to a rapid growth in OTT in India.

With $75 \%$ of Indian internet users in the age group of less than 35 years and more than $50 \%$ of smartphone app users aged between 18 to 24 years, OTT platforms are targeting a younger demographic. (Accenture, 2015)

With 70 million video viewers in India and about 1.3 million OTT paid-video subscribers, the number of Indian consumers inclining towards OTT platforms is increasing rapidly. But, the numbers fluctuate every month. Hotstar claims to have 5 million viewers on a regular basis which could rise up to 100 million during IPL season. (Accenture, 2015).OTT services in India uses top-down approach in India but to be successful in the long term, a bottom-up approach to reach at every segment of internet savvy population.

\section{KNOWLEDGE GAP}

One of the primary things that the literature has pointed out to is the consistent lack of concrete evidences to understand and assimilate the consumption patterns of millennial in India. Although it has shown multiple factors that are leading to the rise of OTT platforms in India, there are hardly any research papers from the consumption viewpoint suggesting which specific content is consumed by which specific target group and the reason for the same. It has also shed light on the factors that have affected the content viewing on OTT platforms, but questions still rise on whether those remain the only factors that affect the same. There is yet a need of a detailed study which describes in detail the kind of preferences that millennial have in terms of the video content and whether or not it will ever lead to the popularity of OTT platforms in India. Thus, the knowledge gap prevalent in the review is the dearth of research to understand reasons for consumer preferences of content on OTT platforms. A lot of research has been done to study the factors contributing to the success of OTT in India but not much has been researched on the factors contributing to millennial consumer adoption of OTT services in India. This research paper tries to understand the factors leading to adoption of OTT services by millennial consumers on a long term. This will help companies offering OTT services an insight into the reasons for consumer preference for this service and ways to induce repeated purchase and final adoption of OTT platforms in India.

\section{CONCEPTUAL FRAMEWORK}

In terms of the conceptual framework, there are some concepts which require special attention in reference to the meaning that they carry with respect to this research paper.

Innovation: Any good, service or idea that is perceived by someone as new. (Keller, 2017)

Adoption: An individual's decision to become a regular user of a product. (Keller, 2017)

Innovation-Diffusion process: The spread of a new idea from its source of invention to its ultimate users or adopters. (Keller, 2017) 


\section{THEORETICAL FRAMEWORK}

\subsection{Innovation Adoption Model (AIETA Model)}

This model developed by Rogers focuses on the mental processes through which a customer passes through from the point he hears about an innovation till he finally adopts it. (Keller, 2017).

Awareness: The consumer comes to know about the innovation but lacks information about it.

Interest: The consumer is motivated to seek information about the innovation.

Evaluation: The consumer considers whether to try the innovation.

Trial" The consumers tries the innovation for the first time.

Adoption: The consumer becomes a regular user of the product.

\subsection{Users and Gratification Theory}

The theory of Uses and gratification aims at explaining the way in which people use media to fulfill their needs and derive gratification out of it. People tend to get interact, receive knowledge, relax, be aware and entertain themselves through the media which they find convenient to use for interpersonal communication as well. Users become an active ingredient to the communication process, being goal oriented in their media use. The theorists suggest that media user look for specific media sources that will best suit their needs and satisfy their gratification. Uses and gratifications assume that users have more than one choices to satisfy their need. This theory unlike other theories holds that users are accountable for selecting media that matches with and satisfies their desires and needs.

\section{RESEARCH QUESTIONS}

The research questions in this case are the following:

1. What triggers millennial to prefer OTT platforms over traditional video viewing platforms like TV?

2. How often is it observed that millennial prefer OTT platforms over TV?

3. What are the typical behavioral, personality traits of the consumers who consume content on OTT platforms?

4. What are the content types that are preferred for these consumers?

\section{RESEARCH DESIGN}

\subsection{Methodology}

The methodology adopted in this research was qualitative in nature. This methodology was chosen as not much literature was available for this research as well as the research required an in-depth understanding of gratification factors by delving deeper into the consumer mind for gathering insights. Interpretative paradigm was taken into consideration. The research adapted an in-depth interview method since the requirements of the research was to use laddering technique and understand the root-cause analysis of consuming content in OTT platform. This method is usually helpful in obtaining reasons and causes through an in-depth conversation on current video viewing patterns, behaviors, beliefs, norms, attitudes, and practices that might have influence on their OTT content usage. The tool of qualitative 
research was a carefully prepared discussion guide consisting of ice breaking, generic and specific questions. The points in the discussion guide helped the interviewer to converge the research to a certain direction. Hence, the researcher undertook a convergent strategy to arrive at the themes of gratification. Thematic content analysis was done based on the transcripts of the interview. From the findings of the themes, inferences were drawn as to the popularity, sustenance and marketable quotient of the medium. The findings and analysis justified the research questions and the objectives of this research.

\subsection{Data collection}

The participant planning for this research was that the participants consisted of young, dynamic, post-graduate individuals, who belong to the generation that is defined as millennial or echo boomers. The target participant was in the age group of 18-30 years belonging to metro cities. The reason for choosing such a target group was the fact that these individuals are frequent users of OTT platform. Hence the participants were carefully chosen so that they consume OTT content on a regular basis or are addicted to OTT content by been consuming video content on one or more platform on a regular basis either through laptops, desktops, or/and on their mobile devices. Most of these participants would choose OTT content over traditional television content and are frequently watching those contents that are customized for OTT platform only. Theoretical sampling procedures were applied and the final participant size of in-depth interviews was 35 individuals. This number was arrived at as there was theoretical saturation after 30 participants and no new information was being generated. The interviews were mostly conducted on a one-on-one basis, however, few interviews were conducted over telephone as well. The sampling method that was used in this case was referential and snowball sampling process. The time taken for each interview was approximately 30 minutes and then the responses were coded. Inter-coder reliability of $90 \%$ was achieved in this research. Funneling technique of interviewing was adopted and points flowed in a logical coherent manner from generic to specific questions. Themes were based on the coding and inferences were drawn from the emerged themes.

\subsection{Findings and Analysis}

The findings of this research suggest relevant themes that emerged for the preferred adoption and consumption of OTT content among millennial. The findings were based on 35 respondents who were interviewed and probed and the questions primarily mapped their usage patterns, content preferences, perception of OTT platforms and did an overall comparison with respect to video consumption patterns of other video media vehicles like television and downloaded content.

\subsection{Adoption factors}

It is an interesting insight that these 35 respondents claim to have come to know of these OTT platforms through either of the two stimulus: mainstream media advertising by promoting the platform on multiple media vehicles or through referrals and word of mouth suggestions through friends, family and relatives. These were the two major sources through which millennial would get knowledge and develop interest for adopting a particular platform or platforms. So the first strategy that any of these branded platforms should focus on, is either putting their brand in different media vehicles and reinforcing the message or developing a strategy to increase word-of-mouth marketing and referral promotions. This research suggests that there are four major reasons why millennial consumers would adopt a certain platform and view its contents. 


\subsubsection{Convenience}

"It is so useful that I can easily navigate through it" says Rajesh Sharma, while talking about an app related to banking. Another respondent, Ritu Jha commented, "I can chat with friends anytime I want through WhatsApp. It is suitable to the format of conversing and exchanging information". Similarly, many respondents in this study claimed that the kind of ease with which they are able to use and adapt to these platforms are much higher as compared to the amount of clutter they end up dealing with on the loads of channels on television. They suggested that in a fast moving consumer world, where time is a scarcity, if apps and different OTT platforms provide convenience in terms of banking, shopping, making travel plans, organizing events etc. then it will be a much sought after platform. However, some of the respondents were also of the opinion that navigation through these platforms need to be made easy and less time consuming. This facility will gratify consumers with the objective of solving more work in less time. Hence, it can be inferred that one of the major reasons of adoption of a certain OTT platform is based on the convenience that the platform provides. The marketers or the app developers need to take into consideration who is app is being made for and for what purpose. These two questions need to be kept in mind as different target groups have different perceptions about convenience. So, if the target group and the purpose of the target group in using in app is clearly understood by the brand, the OTT platform brand should work to enable these facilities and communicate it to the target group.

\subsubsection{Mobility}

Another major reason for adoption of OTT platform is the freedom of movement that these platforms provide. "I wanted to watch a cricket match while travelling. Thank God, I had Hotstar installed" says Rakshit Menon, an entrepreneur who loves cricket but at the same time has to travel a lot. Mobility is thus an important adoption factor for the OTT brands. With so much of corporate tours that employees need to do, it becomes difficult to watch television or any static medium. The requirement of the hour is something "on-the-go". OTT platforms gives flexibility to the viewer to watch any programme wherever he/ she goes. So the content can be watched anytime at any place. The consumer is ready to pay additional cost provided the consumer gets freedom and flexibility to watch anything anywhere. According to Mohan Nath, a budding journalist, "I need to be aware of the news every time I travel. OTT platforms give me that liberty to be constantly updated with the latest happenings." Hence the second factor that the communicators of OTT needs to keep in mind is to express the benefits of OTT platform and content in terms of the freedom and mobility it provides to watch anything at any time without missing even if it has already been telecasted. Hence delayed telecast is gaining a lot of popularity as it is not only convenient to watch, but also through OTT platforms, it is more mobile in nature. For enticing customers to avail the OTT brand, the marketing should be done in such a way that the benefit fits into the enhanced mobility need of the customer.

\subsubsection{Content}

Among the four parameters of adoption strategy, content occupies the central position in terms of interest generation among the audience. "If the content is not good, no matter how the convenience of a platform is, I will not be interested to visit again", says Ranjit Gupta, a student of a reputed college. Almost all respondents were of the opinion that all other factors aside, it was the kind of content that attracted them to use these platforms in the first place after being introduced to it. Hence, it is very important to put in the appropriate interesting content in these platforms. The content, according to respondents, need to be contemporary, 
interactive and fun to engage with. There could be graphic interfaces and innovative visual effects need to be incorporated so that it entices the millennial consumers to be engaged, involved and interact with the content. In this way, there will be enhanced gratification of the platform. So, it can be inferred that there if the content is not engaging, other marketing elements will not be able to popularize the platform, given the fact that there is intense competition between these platforms. The brand needs to cater to attractive content and genre to connect to the audience. The millennial audience is extremely choosy and flirtatious with different platforms. The contemporariness of the content is something that brand managers and platform developers need to concentrate on so that there are repeat viewing of the platform. This is possible when the theme of the OTT platform, the audience and the content is in sync with each other and creates an effective connect among themselves. This process will ensure that consumer connect is optimum in these platforms in the cluttered OTT platform industry.

\subsubsection{Cost}

OTT video platforms have a peculiar problem especially in a market like India. The concept of a paid subscription is still quite nascent in the market and thus most people are still restricting themselves to either free content or limited free period that the brand/platform offers. Out of the 35 respondents only 10 respondents were subscribed to or have ever paid for a premium subscription on an OTT platform from the time they started using them. Most respondents were of the opinion that they would rather enjoy themselves in these platforms free of cost. However, when money is involved to access these platforms, respondents were slightly hesitant to pay for such platforms. Only 12 out of 35 respondents showed any willingness to pay for the subscription of an OTT platform. The challenge also lies in the fact that many OTT platforms in the process of giving a tough competition to other brands end up giving premium content at no cost for the customer and thus end up losing their revenues in order to win the customer over. However, some of the respondents did mention that if they changed their consumption pattern from television to OTT, then they would not only be able to watch the content of their choice and the time of their preference, but they would also be paying lesser amount of subscription than what they would pay for the television. This insight is in fact interesting, that television as a consumption medium is basically a wastage of money in terms of the amount paid to cable operators and the redundancy of the plethora of channels present in the television. The television offers around thousand channels in some operators and the price that has to be paid is high as the whole package has to be taken and hence television becomes an expensive medium. However, OTT platforms are customized and hence the viewer pays exactly what the viewer wants to pay and not any additional cost of any channel for which the viewer is not interested.

The competition of the OTT platforms is thus not only among themselves but also with other media for that matter. As a result, the marketers of OTT platform needs to educate the audience that they are actually paying for what they are watching in terms of the OTT platform but in terms of television, they are paying even for what they are not watching and hence television is an expensive medium in comparison with the OTT platforms. Consumers, till date are more attracted to the free OTT platforms than the paid ones because their perception is that OTT is an additional entertainment medium. The moment consumers are made aware that OTT platform is not supplementary but complimentary to television, then their perception towards cost of paying for the medium will change. Consumers need to be educated that OTT platform comes as a replacement to their television viewing because the consumers get to watch exactly what they want and pay exactly what they watch. This process of awareness would slowly change the mindset of the consumers and they would start switching to more paid platforms than the free ones. This process may in the future lead to 
lesser viewing of television but more effective viewing of OTT platforms in terms of investment by a consumer for entertainment expenditure.

These are the major four factors that may lead to a certain consumer adopt this new medium of OTT branded platforms or be indifferent towards the platform. Marketers and brand managers need to concentrate in these four areas to create traction about these platforms and their brands.

\section{CONCLUSIONS}

This research is a novel attempt to not only discuss how consumers will adopt to a certain new meta-medium known as the OTT platform but to also understand its repercussions in terms of what is in store for the future in terms of media consumption. In other words, if customers adopt to the OTT format, what will its consequences be in terms of television viewing. The reason of comparing television with OTT platform is that both are audio-visual in nature, the usage of both media are similar and both media provide similar gratification. While comparing OTT versus television, all 35 respondents said that their television consumption patterns have been reduced to being minimal post introduction of OTT platforms. 25 out of 35 respondents claimed that OTT platforms were much better than traditional video consumption media vehicles like television.

With the internet and mobile penetration increasing by the day, the future of OTT looks bright. Although people have started investing a lot of time and money on OTT platforms, it is difficult to say that it will ever replace television as a primary video consumption platform. The pricing strategies of almost all OTT platforms is currently at a substantially higher side for the Indian consumer and thus is leading to a high dissuasion by them towards becoming a regularly paying customer. Despite having tie ups with multiple brands and gaining and attracting multiple brands to collaborate with the platforms, OTT channels are finding it extremely difficult to maintain a steady streamline of revenue and thus are highly dependent on paying subscribers for their revenues. Data consumption is one of the key concerns that millennial have. Although with tough competition being given by multiple telecom providers in terms of data plans, the consumption levels of data on OTT platforms remains similar and thus making no substantial difference to the overall cost that the end consumer ends up bearing to view the content on the platform. It was also realized the millennial specifically are on the lookout in specific genres that suit their need and do not appreciate replication of content especially if it is available already on the internet or traditional channels like television. Thus OTT platforms must always be on a lookout for avenues to create and provide more attractive and highly engaging content that isn't available elsewhere. The unfortunate problem in this case is that not all OTT platforms have the financial capabilities to pursue new production of video content, thus making it almost next to impossible for newer and smaller OTT platforms to create and generate newer content that specifically caters only to OTT platforms and devices.

The implications are significant for marketers from this paper. Marketers need to communicate to the consumers that OTT platforms are a part of their convenience need which has become part and parcel of everyday life. On top of that, consumers are free to watch any content anytime and anywhere. However, special care has to be taken not only to stream content but to create and innovate content. In today's world, content procurement is easily available and hence newer content needs a lot more creativity and engagement visuals. Finally, the subscription cost is another hindrance to the free flow of this platform and most people prefer free videos than paid videos. The marketer needs to communicate that their brand provides maximum convenience, portable and movable anywhere, extremely contemporary, interactive and useful in terms of content and finally the consumer is paying 
what the consumer is watching and no additional payment is done unlike other media. This awareness will lead to higher levels of equity of the OTT platform with the consumer. However as a fallout of more adoption of OTT platform, television may lose a bit of customer base as OTT platforms are more related to the lifestyle of the millennial. Television may redefine another target audience as niche leaving the millennial consumers to the instant onthe-go medium like OTT.

In conclusion, there is a huge need to streamline the entire process of OTT market in India. Content makers and technology providers need to specifically focus on creating a platform which can cut through the clutter and make all of the available video in an integrated fashion. On the other hand, users and subscribers also need to understand that it may not always be possible for the OTT platforms to end up providing an integrated video content platform for free and that it is for providing a better viewing experience that the OTT platforms function the way they do in its current fashion.

\subsection{Limitations of Research}

In terms of the limitations of this research, there were four major limitations: firstly, there was a huge dearth of existing literature on OTT platforms, its usage and experience throughout the literature. Secondly, paucity of time and resources meant that convenience sampling had to be undertaken by the researcher, which led to respondents being fairly homogenous in nature demographically, and also being sourced from only two cities - Pune and Mumbai. Finally, the fact that OTT platforms are still a nascent concept in India meant that the researcher could not cast a wider net as far the number of respondents were concerned.

\subsection{Scope of Future Research}

This research currently only covers the consumer's point of view in terms of the consumption of video on the OTT platforms, thus not taking in to picture at all the channel and the platforms point of view. Further research must be conducted with different stakeholders, target audiences and experts in order to understand and integrate the brand's point of view to gain a more cohesive and holistic view on OTT platforms.

\section{REFERENCES}

[1] Accenture. Digital Video \& the connected consumer. Accenture. Retrieved Oct 15, 2017, from https://www.accenture.com/_acnmedia/Accenture/ConversionAssets/Microsites/Documents17/Accenture-Digital-Video-Connected-Consumer.pdf (2015).

[2] Deloitte. Digital Media: Rise of on-demand content. Deloitte. Retrieved Oct. 15, 2017, from https://www2.deloitte.com/content/dam/Deloitte/in/Documents/technology-mediatelecommunications/in-tmt-rise-of-on-demand-content.pdf (2017).

[3] Digital Media-Rise of on-demand content. Deloitte. (2015).

[4] Ernst \& Young. Future of Digital Content Consumption in India. Ernst \& Young. Retrieved from http://www.ey.com/Publication/vwLUAssets/ey-future-of-digital-january2016/\$FILE/ey-future-of-digital-january-2016.pdf (2016).

[5] IAMAI. Internet and Mobile Association of India. IAMAI. Retrieved Oct 15 (2017)

[6] Keller, P. K. Marketing Management. Pearson. (2017).

[7] KPMG-FICCI. Indian Media \& Entertainment Industry. KPMG. Retrieved oct. 14, 2017, from https://assets.kpmg.com/content/dam/kpmg/pdf/2015/03/FICCI-KPMG_2015.pdf (2015).

[8] Lacy, L. Digital marketing spend to hit $\$ 118$ bn by 2021 , but budgets will shift to experiences. The Drum. Retrieved Oct 15, 2017 
[9] OTT video market update. Frost \& Sullivan. (2016).

[10] Statista. (n.d.). Statista. The Statistics Portal. Retrieved Oct 16, 2017, from https://www.statista.com/topics/2157/internet-usage-in-india/

[11] The Future: Now Streaming. FICCI-KPMG. (2016).

[12] The Future of Internet in India. NASSCOM. (2016).

[13] Video OTT vs DTH players: How Netflix, Amazon Prime, Hotstar cornered Indian market. (2017, November 2017). Financial Express. Retrieved Oct 16, 2017, from http://www.financialexpress.com/industry/video-ott-vs-dth-players-how-netflix-amazonprime-hotstar-cornered-indian-market/948816/ 